\title{
On Extracting User-centric Knowledge for Personalised Quality of Service in 5G Networks
}

\author{
J. Sánchez-González, O. Sallent, J. Pérez-Romero, R. Agustí \\ Dept. of Signal Theory and Communications \\ Universitat Politècnica de Catalunya (UPC) \\ Barcelona, Spain \\ e-mail:[juansanchez,sallent,jorperez,ramon]@tsc.upc.edu
}

\begin{abstract}
This paper aims to improve the user Quality of Service (QoS) in 5G networks by introducing a user-centric view that exploits the predictability of the user's daily motifs. An agglomerative clustering is used to identify these motifs according to the cells in which the user is camping during the day. Then, a technique to extract the personalised QoS observed by the user is proposed. The methodology is illustrated with an example that makes use of real measurements obtained from a specific customer of a $3 G / 4 G$ operator. The presented results illustrate that the proposed user-centric approach is able to identify situations with poor user perceived QoS which could not be identified by a classical network-centric approach.
\end{abstract}

Keywords- User-centric optimization, knowledge discovery, daily motifs, clustering.

\section{INTRODUCTION}

As a next step in the evolution of mobile communication systems, research carried out by industry and academia is nowadays focused on the development of the new generation of mobile and wireless systems, known as the 5th Generation (5G). Requirements for future $5 \mathrm{G}$ system have already been identified and discussed in different fora [1][2]. The vision of the future $5 \mathrm{G}$ corresponds to a highly heterogeneous network at different levels, including multiple Radio Access Technologies (RATs), multiple cell layers, multiple spectrum bands, multiple types of devices and services, etc.

$5 \mathrm{G}$ is envisioned to include a higher degree of automation in the planning and optimization processes through the introduction of Self Organizing Networks (SON) functionalities [3], so that operating costs can be reduced and human errors minimized. While the current SON vision exhibits an intrinsic reactive design approach and a lack of endto-end knowledge of the network [4], the inclusion of Artificial Intelligence (AI)-based tools enables to shift the evolution of the SON paradigm in $5 \mathrm{G}$ towards a more proactive approach incorporating additional dimensions coming from the characterization of end-user experience and end-user behavior. Specifically, [5] presents a framework that processes input data from multiple sources and extracts, through learning-based classification, prediction and clustering models, relevant knowledge to drive the decisions made by $5 \mathrm{G}$ SON functionalities. Given the ultra-high level of efficiency associated to the design of future $5 \mathrm{G}$ systems, the target is to gain in-depth and detailed knowledge about the whole ecosystem, understanding hidden patterns, data structures and

This work has been supported by the EU funded H2020 5G-PPP project SESAME under the grant agreement 671596 and by the Spanish Research Council and FEDER funds under RAMSES grant (ref. TEC2013-41698-R). relationships, which in turn will enable ultra-efficient management and optimization. Furthermore, big data analytics [6] appear as an enabling technology for the management and operation of $5 \mathrm{G}$ networks. Network data is becoming both an important issue and an opportunity for operators to be able to fully exploit the great value in this data.

On the one hand, as their core business matures and markets reach saturation, MNOs need new revenue streams. In this respect, MNOs could package anonymous versions of this data and sell it to businesses in retail, travel, and other consumer-facing industries; these businesses, in turn, could use the data to refine their customer understanding and improve their marketing efforts through behavioral targeting, personalized marketing, targeted location-based ads, and other services. MNOs could also use this data to offer services to their own retail customers, enabling them to access services from Over-The-Top (OTT) players from within the security of the operator's ecosystem [7]. So far, the usage of call detailed record data, collecting the time and the base station of the mobile terminal communication activity (messages, calls, etc.), has been used for urban and transportation planning purposes, targeting to identify daily motifs given that human daily mobility can be highly structural and organized by a few activities essential to life [10].

On the other hand, the higher level of knowledge about the network and its users constitutes a key differential factor between 5G networks and legacy systems. According to [8], network coverage, voice quality and Internet quality impact on customer retention, so these are key factors in customers' decisions to stay with their current operator or churn to another operator. Therefore, MNO's service operation and management needs to be redefined to put customers at the heart of daily operations. In this sense, MNOs need a complete and real-time picture of service behavior and performance for each individual customer. Hence, a challenge for $5 \mathrm{G}$ is to support applications and services with an optimal and consistent level of QoS anywhere and anytime. Too low QoS leads to user dissatisfaction, whereas too high QoS unnecessarily drains resources on both the user (e.g., device battery) and operator (e.g., network resources, base station power) sides [9]. Regarding the exploitation of network data for MNO's internal processes, [5] envisages several components to have high interest and applicability (e.g., spectrum planning, inter-cell interference coordination, optimization of admission control, energy saving) for the user-level characterization.

In this context, a personalized QoS assessment model is devised in this paper exploiting the fact that human daily 
motifs show a high degree of temporal and spatial regularity. After analyzing the similarities encountered in the daily individual travel patterns of a user through a clustering approach, some relevant QoS parameters can be extracted automatically, paving the way for an operator to make the most of this user knowledge. In particular, a clustering technique is used in Section II to determine the daily motifs of a given user in relation to the observed serving cells. Then, a technique for characterizing the Quality of Service (QoS) obtained by the user based on the previously obtained daily motifs is proposed in Section III. Finally, conclusions are drawn in Section IV.

\section{USER-LEVEL KNOWLEDGE DISCOVERY: DAILY MOTIFS}

\section{A. Clustering methodology}

The proposed methodology aims to process the measurements carried out by a given User Equipment (UE) in order to extract a set of mobility patterns that capture the daily motifs of this UE. This is done by sampling and recording the Physical Cell Identifier (PCI) of the serving cell for a given UE during several days, weeks or even months. Since the obtained number of samples will typically be a large value (e.g. UE measurements might be collected every few seconds), a preprocessing step is necessary in order to obtain vectors of shorter dimension. For this purpose, the measurement reports collected during a specific day $d$ (with $d=1, \ldots, D$ ) are grouped in $M$ periods of time with equal duration $T$. Let define the vectors $\boldsymbol{C}_{d}=\left\{c_{d, 1}, c_{d, 2}, \ldots, c_{d, M}\right\}$ so that the term $c_{d, m}(m=1, \ldots, M)$ represents the PCI of the cell where the UE is camping on during the $m$-th time period of the $d$-th day (i.e. for a UE in connected mode this will be the serving cell, while for a UE in idle mode it will be the cell where the UE has camped on after the cell selection/reselection procedures $)^{1}$. In case that, in a particular period of time $m$, there exist more than one serving cell, then, $c_{d, m}$ is set to 0 . This would correspond to a time period in which the UE has been moving across multiple cells.

The characterization of the UE daily motif is done by means of a clustering process that makes use of the vectors $\boldsymbol{C}_{\boldsymbol{d}}$ and groups them into clusters in a way that days of the same cluster are similar among them and different from the days of the rest of the clusters. In this paper, the clustering process is done by means of an agglomerative clustering [1]. The agglomerative clustering belongs to the family of hierarchical clustering methodologies which do not assume a priori the number of clusters, but build a hierarchy tree with different clustering levels. The agglomerative clustering algorithm operates iteratively. In the first iteration, the algorithm starts at the lowest clustering level where each of the days (i.e. each vector $\boldsymbol{C}_{\boldsymbol{d}}$ ) is considered as a cluster itself. Then, the algorithm merges the two clusters with highest similarity (i.e. lowest dissimilarity) into a single one. This operation is repeated iteratively until all the days are grouped in a single cluster. The result is represented by a dendrogram that consists on a tree diagram that illustrates the different levels of clustering.

In this paper, the dissimilarity $L$ between two clusters is measured as the average value of the Hamming distance

\footnotetext{
${ }^{1}$ For UEs in connected mode, the collection of measurements can be done using the RRC Measurement Reports transmitted by the UEs to the network. For UEs in idle mode, the collection can be done through the Minimization of Drive Tests (MDT) feature [12], which gives the possibility that UEs collect measurements in idle mode and transmit them when they connect to the network.
}

between all the days of one cluster and all the days of the other cluster [1]. The Hamming distance between two days represented by vectors $\boldsymbol{C}_{\boldsymbol{i}}=\left\{c_{i, 1}, c_{i, 2}, \ldots, c_{i, M}\right\}$ and $\boldsymbol{C}_{j}=\left\{c_{j, 1}\right.$, $\left.c_{j, 2}, \ldots, c_{j, M}\right\}$ is determined as the number of elements (time periods of the day) in which these vectors differ (i.e. $c_{i, m} \neq c_{j, m}$ with $m=1, \ldots, M)$. By cutting the resulting dendrogram at a certain dissimilarity level threshold $L_{t h}$ a set of $K$ clusters is obtained. Each $k$-th cluster consists on a set of $N_{k}$ days that are clustered together. For each of the $K$ obtained clusters, a prototype $\boldsymbol{U}_{\boldsymbol{k}}=\left\{u_{k, 1}, u_{k, 2}, \ldots, u_{k, M}\right\}$ with $k=1, \ldots, K$ is defined in order to represent all the days that belong to the $k$-th cluster. The vector $\boldsymbol{U}_{\boldsymbol{k}}$ is obtained so that each term $u_{k, m}$ is calculated as the mode (i.e. the most frequent value) among the $c_{k, m}$ values of the days that belong to the $k$-th cluster. Additionally, two statistical indicators are also included for each cluster:

- Percentage of hits $\left(A_{k}=N_{k} / D\right)$ : It is the percentage of days that belong to the $k$-th cluster. The prototype of clusters with high value of $A_{k}$ will be more frequent and representative.

- Average Hamming distance of the days that belong to the $k$ th cluster $\left(H_{k}\right)$ : It is the dissimilarity between the different days of the cluster with respect to the prototype $\boldsymbol{U}_{\boldsymbol{k}}$ of the cluster. A cluster with a low value of $H_{k}$ reflects a lower dispersion in the cluster, meaning that the prototype is more representative of the days that belong to the cluster.

\section{B. Applicability example}

In order to illustrate the behavior of the proposed methodology, measurements from a specific customer of a $3 \mathrm{G} / 4 \mathrm{G}$ operator in Spain have been collected initially during $D=14$ days. For each $d$-th day, the vector $\boldsymbol{C}_{\boldsymbol{d}}$ has been built using $M=96$ time periods of $T=15$ minutes each. The agglomerative clustering has been implemented by means of RapidMiner Studio [14]. The obtained dendrogram is represented in Figure 1. The horizontal axis presents the dates of the $D=14$ days while the vertical axis presents the cluster dissimilarity $L$. By cutting the dendrogram with a threshold $L_{t h}$ a number of clusters are obtained. For $L_{t h}=25, K=2$ clusters have been identified as shown in Figure 1. It is observed that Cluster 1 is formed by three weekend days, while Cluster 2 contains all the days from Monday to Thursday. Finally, there are three days that are neither clustered in Cluster 1 nor in Cluster 2 because the cell connectivity pattern of the UE during these days was considerably different than the rest of the days. The percentage of hits and the average Hamming distance of the two clusters are $\mathrm{A}_{1}=21.42 \%, \mathrm{~A}_{2}=57.14 \%, \mathrm{H}_{1}=6.66$ and $\mathrm{H}_{2}=8$, respectively. The high values of $A_{1}$ and $A_{2}$ indicate that most of the days belong to any of the two identified clusters, while the low values of $H_{1}$ and $H_{2}$ indicate that the obtained prototypes are very representative of the days that belong to each cluster. The prototypes are represented in Figure 2. Two daily motifs can be inferred for this user: (1) From Monday to Thursday the user goes from home $(\mathrm{PCI}=191)$ to work $(\mathrm{PCI}=363)$ and back home, as reflected by cluster 2, and (2) The user spends most of the weekend at a different home $(\mathrm{PCI}=60)$, as reflected by cluster 1 . The obtained prototypes enable the definition of a state diagram representing the daily motifs, as shown in Figure 2.

To illustrate the sensitivity of the proposed methodology to the measurement collection period, the UE measurements have 


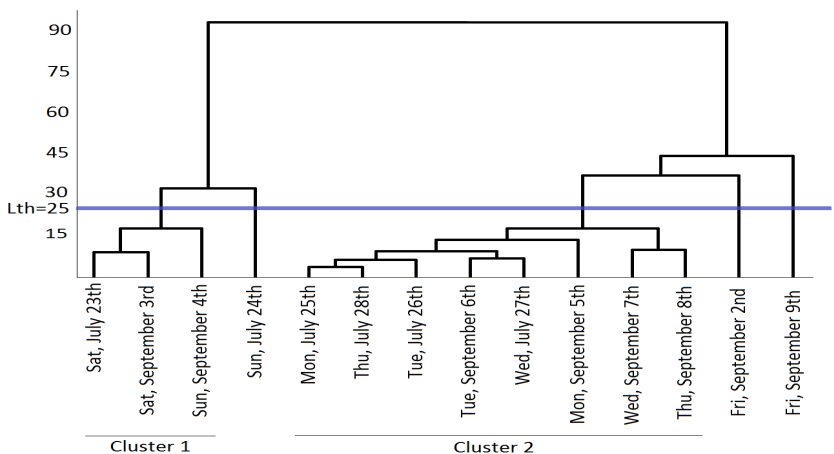

Figure 1.- Obtained dendrogram for $D=14$ days.
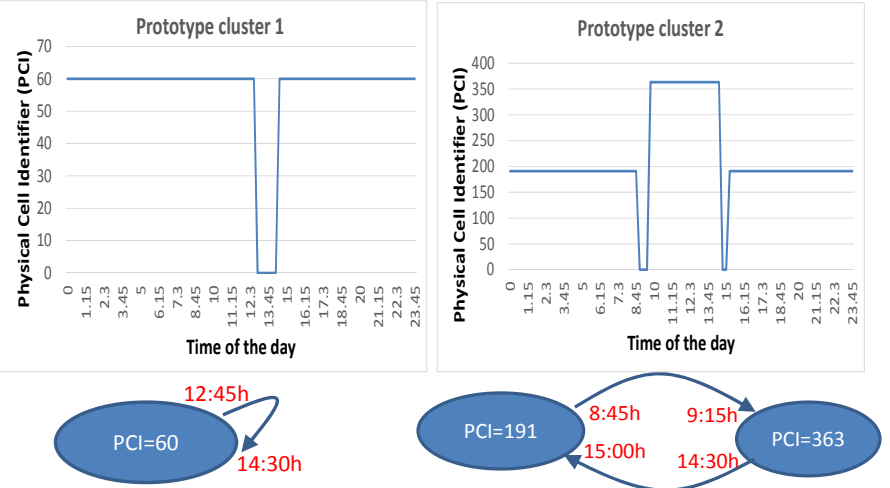

Figure 2.- Prototype vectors and state diagrams of the $K=2$ clusters.

been extended for a higher number of days (i.e. $D=77$ days). Figure 3a presents the percentage of hits $A_{k}$ while Figure $3 \mathrm{~b}$ represents the average Hamming distance $H_{k}$ of the days that belong to each cluster with respect to the prototype of the cluster. Four representative clusters (i.e. clusters 1, 2, 3 and 4) can be identified with a relatively high percentage of hits as shown in Figure 3a. The low value of the average Hamming distance seen in Figure $3 b$ for these four clusters indicates that the prototypes are very representative of the days that belong to each cluster. On the other hand, four small clusters (i.e. clusters 5, 6, 7 and 8) are not considered as representative since each of them only represents $2.5 \%$ of the total number of days, as shown in Figure 3a. Finally, there are ten days that have not been grouped in any of the identified clusters since the cell connectivity pattern of the UE during these days was considerably different from the rest of the days. The state diagrams of the four representative clusters are shown in Figure 4. Four daily motifs for this user can be inferred: (1) Some weekends the UE mainly stays at home $(\mathrm{PCI}=191)$, as captured by cluster 2. (2) Some weekends the UE mainly stays at a weekend's countryhome ( $\mathrm{PCI}=60$ ), as captured by cluster 1 . (3) Some working days, as captured by cluster 3 the UE goes from home (PCI=191) to work ( $\mathrm{PCI}=363)$ and back home. (4) Several working days, as captured by cluster 4 , the UE goes from home $(\mathrm{PCI}=191)$ to a company's venue $(\mathrm{PCI}=363)$, then the UE goes back home to have lunch $(\mathrm{PCI}=191)$, the UE goes to work to another company's venue $(\mathrm{PCI}=330)$ in the afternoon and goes back home at night $(\mathrm{PCI}=191)$. The days that do not belong to one of these four clusters correspond to non-frequent habits of the user, for example, days when the UE stayed at night at the weekend's home $(\mathrm{PCI}=60)$ and went to work to the company's venue ( $\mathrm{PCI}=363$ ) or some working days in which the user did not go to the office due to different reasons (e.g. business trips), etc.

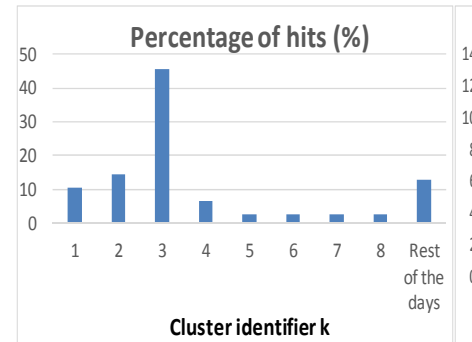

(a)

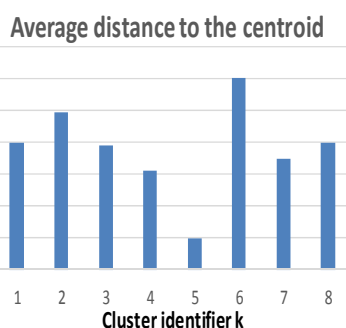

(b)
Figure 3.- Percentage of hits and average Hamming distance.

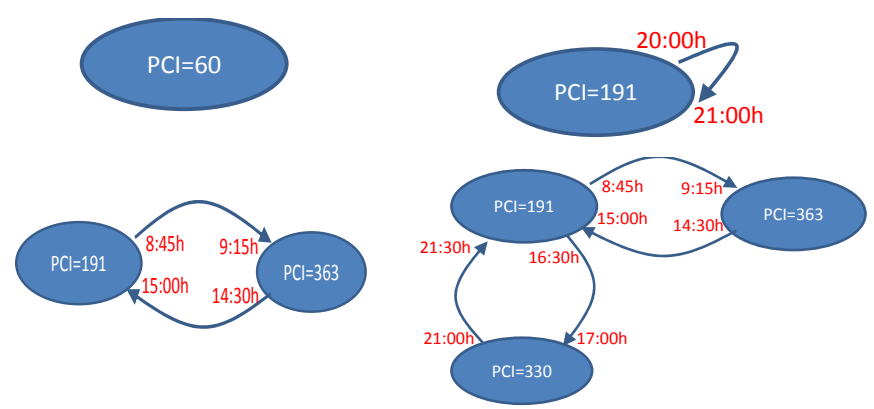

Figure 4.- State diagrams for $\mathrm{D}=77$ days for prototypes $\mathrm{k}=1$ (top-left) to $\mathrm{k}=4$ (bottom-right)

\section{USER-LEVEL KNOWLEDGE DISCOVERY: QUALITY OF SERVICE}

\section{A. From network-centric to user-centric KPIs}

The performance monitoring carried out nowadays by MNOs typically follows a network-centric view, where the MNO collects per-cell KPIs (e.g., call dropping rate, average or cell-edge throughput) and establishes thresholds in order to raise underperformance alarms. Radio network optimization engineering teams rank the affected cells and attempt to tune network parameters to improve the performance. The advent of SON capabilities introduces automatization into these processes and alleviates the need for deep and difficult measurements analysis. The advent of Artificial Intelligence capabilities (e.g., learning, prediction, clustering) enables more proactive and advanced approaches. In addition to the above evolutions, introducing a user-centric approach involves a paradigm change. Clearly, the monitoring and analysis of network-centric KPIs such as the average throughput, the $95 \%$ percentile throughput or the cell-edge throughput are adequate to assess whether optimization actions are needed at a specific cell. However, from a user-centric perspective, the above KPIs do not suffice. A specific customer does not mind that the network as a whole is performing adequately if he perceives a non-satisfactory performance at the usual locations where this customer connects to the network. Therefore, a user-centric QoS management requires new metrics and methodologies, as discussed in the next subsection.

\section{B. QoS recording methodology.}

At the same time that the user-level Knowledge Discovery collects the PCI of the serving cell for a given UE in order to capture its daily motifs, the periods where services are activated and the corresponding QoS achieved can also be recorded. As a result of the clustering process in Section II, $K$ 
prototypes are defined. Each prototype has an associated state diagram (see e.g., Figure 4). Let denote that the $k$-th prototype has $M_{k}$ states. Each state of each prototype has associated service-usage patterns (e.g., probability that a given service is activated) as well as QoS performance metrics (e.g., average throughput observed, peak throughput observed). Therefore, knowledge about what, where and when the UE activates each type of service together with the observed QoS is retained.

The MNO has the possibility to exploit the proposed usercentric view pursuing increasing levels of detail for the QoS provisioning: (i) an overall QoS metric (i.e., a weighted average of the QoS observed by the UE in the different prototypes), (ii) a per-prototype overall QoS metric (i.e., a weighted average of the QoS observed by the UE in the different states of the prototype) or (iii) a per-state and perprototype QoS metric. Specific actions to improve the QoS for this UE would be triggered if the merits of these metrics are below certain thresholds. The service usage is defined as the fraction of time in which the user is in connected mode and it is defined at an overall, a per-prototype and a per-state level. In turn, the per-state QoS metric is computed as the average value of the QoS metric when the user is in connected mode at a given state. The per-prototype QoS metric is obtained by averaging the corresponding per-state QoS metric weighted by the service usage of each state of the prototype. Finally, the overall QoS metrics are measured as the average per-prototype metrics weighted by the per-prototype service usage.

\section{Applicability example}

In order to illustrate the proposed methodology, the periods of time when the user is in connected mode have been recorded to determine the service-usage patterns during the $D=77$ days measurement campaign leading to the prototypes and state diagrams depicted in Figure 4. As QoS metrics, the uplink and downlink throughput have been measured in the periods of time in which the UE is in connected mode. Table 1 shows the overall, the per-prototype and the per-state and perprototype results in terms of service usage, uplink and downlink throughput. A relatively high service usage is observed for prototype $k=1$ compared to the rest of prototypes.

Let assume that the MNO considers satisfactory an average uplink throughput above $10 \mathrm{Mb} / \mathrm{s}$ and a downlink throughput above $20 \mathrm{Mb} / \mathrm{s}$. According to Table 1, the overall uplink throughput observed by this particular UE wouldn't be satisfactory, while the overall downlink throughput would be good enough. Therefore, if the MNO is only concerned about the overall QoS observed by this UE, only actions to improve the uplink performance will be undertaken. Nevertheless, if the MNO exploits the UE-centric view at a higher level of detail, the per-prototype analysis indicates that the UE also experiences poor downlink performance for prototype $k=1$. This means that, while during working days the downlink performance is good enough, the user experiences poor performance during weekends (when e.g., the UE moves to a countryside home). Therefore, if the MNO is concerned about improving the QoS of this UE, actions to improve the downlink performance at cell $\mathrm{PCI}=60$ will be undertaken.
Table 1.- Per-prototype and per-state QoS metrics.

\begin{tabular}{|c|c|c|c|c|c|c|c|c|}
\hline & & $\begin{array}{c}\text { Prot. } \\
\mathrm{k}=1\end{array}$ & $\begin{array}{l}\text { Prot. } \\
\mathrm{k}=2\end{array}$ & \multicolumn{2}{|c|}{$\begin{array}{c}\text { Prototype } \\
\mathrm{k}=3\end{array}$} & \multicolumn{3}{|c|}{$\begin{array}{c}\text { Prototype } \\
k=4\end{array}$} \\
\hline & & $\begin{array}{l}\text { State 1 } \\
\mathrm{PCI}=60\end{array}$ & \begin{tabular}{|l|} 
State 1 \\
PCI $=191$
\end{tabular} & \begin{tabular}{|l|} 
State 1 \\
$\mathrm{PCI}=191$
\end{tabular} & \begin{tabular}{|l} 
State 2 \\
$\mathrm{PCI}=363$
\end{tabular} & \begin{tabular}{|l|} 
State 1 \\
PCI $=191$
\end{tabular} & $\begin{array}{l}\text { State 2 } \\
\mathrm{PCI}=363\end{array}$ & \begin{tabular}{|l} 
State 3 \\
$\mathrm{PCI}=330$
\end{tabular} \\
\hline \multirow[t]{3}{*}{$\begin{array}{l}\text { Service } \\
\text { usage }\end{array}$} & $\begin{array}{l}\text { Per-state \& } \\
\text { per-prot. }\end{array}$ & 0.0447 & 0.0033 & 0.0112 & 0.0100 & 0.0096 & 0.0011 & 0.0010 \\
\hline & Per-prot. & 0.0447 & 0.0033 & \multicolumn{2}{|c|}{0.0105} & \multicolumn{3}{|c|}{0.0076} \\
\hline & Overall & \multicolumn{7}{|c|}{0.0135} \\
\hline \multirow{3}{*}{$\begin{array}{l}\text { UL } \\
\text { Thr. } \\
(\mathrm{Mbit} / \mathrm{s})\end{array}$} & $\begin{array}{l}\text { Per-state \& } \\
\text { per-prot. }\end{array}$ & 4.12 & 16.73 & 16.27 & 12.83 & 15.29 & 8.72 & 6.15 \\
\hline & Per-prot. & 4.12 & 16.73 & \multicolumn{2}{|c|}{14.64} & \multicolumn{3}{|c|}{13.89} \\
\hline & Overall & \multicolumn{7}{|c|}{7.54} \\
\hline \multirow{3}{*}{$\begin{array}{l}\text { DL } \\
\text { Thr. } \\
(\text { Mbit/s) }\end{array}$} & $\begin{array}{l}\text { Per-state \& } \\
\text { per-prot. }\end{array}$ & 18.66 & 30.80 & 28.78 & 23.72 & 24.7 & 21.14 & 20.82 \\
\hline & Per-prot. & 18.66 & 30.80 & \multicolumn{2}{|c|}{26.39} & \multicolumn{3}{|c|}{24.03} \\
\hline & Overall & \multicolumn{7}{|c|}{21.11} \\
\hline
\end{tabular}

\section{CONCLUSIONS}

This paper has presented a user-centric methodology for characterizing the QoS of individual users by exploiting the predictability of the user daily motifs. An agglomerative clustering technique has been used to identify these motifs and characterize them by a set of prototypes, each of them associated to a state diagram that specifies the serving cells seen by the user in different periods of time as well as the QoS experienced in each state. This enables the extraction of the user QoS with different levels of detail, namely overall QoS, per-prototype QoS and per-state per-prototype QoS. The proposed methodology has been illustrated using real measurements obtained from a specific customer of a $3 \mathrm{G} / 4 \mathrm{G}$ operator. Results have shown that the user-centric approach allows the operator to better identify situations with poor user performance that couldn't be detected by classical network centric optimization mechanisms relying on aggregate statistics for all the users of a cell.

\section{REFERENCES}

[1] METIS 2020 project, http://www.metis2020.com

[2] R. El Hattachi, J. Erfanian (editors) "NGMN 5G White Paper", NGMN Alliance, February, 2015

[3] J. Ramiro, K. Hamied, Self-Organizing Networks. Self-planning, selfoptimization and self-healing for GSM, UMTS and LTE, Wiley, 2012.

[4] A. Imran, A. Zoha, A. Abu-Dayya, "Challenges in 5G:How to Empower SON with Big Data for Enabling 5G”, IEEE Network, Nov., 2014.

[5] J. Pérez-Romero, O. Sallent, R. Ferrús, R. Agustí, "Knowledge-based 5G Radio Access Network Planning and Optimization", ISWCS, 2016,

[6] C-L I., Y. Liu, S. Han, S. Wang, G. Liu, "On Big Data Analytics for Greener and Softer RAN”, IEEE Access, August, 2015.

[7] Enabling the OTT revolution. How telecom operators can stake their claim. Booz \& Company, 2013.

[8] Nokia Siemens Networks 2013 Acquisition and Retention Study

[9] P Agyapong, M Iwamura, D Staehle, W Kiess, A Benjebbour, "Design considerations for a 5G network architecture", IEEE. Com. Mag. (2014)

[10] S. Jiang, J. Ferreira Jr., M. C. González, “Activity-Based Human Mobility Patterns Inferred from Mobile Phone Data: A Case Study of Singapore", IEEE Transactions on Big Data, 2015.

[11] J. F. Monserrat, I. Alepuz, et al., "Towards user-centric operation in 5G networks", EURASIP Journal on Wireless Comm. and Net., Jan. 2016.

[12] Hapsari, W.A., Umesh, A., Iwamura, M., Tomala, M: Minimization of drive tests solution in 3GPP. IEEE Commun. Mag. (2012)

[13] Hastie, T., R. Tibshirani, and J. Friedman, The Elements of Statistical Learning. 2009, Springer: New York. p. 520-528.

[14] RapidMiner Studio: http://www.rapidminer.com 